\title{
Ictiofauna do estuário de São Caetano de Odivelas e Vigia (Pará, Estuário Amazônico)
}

\author{
Daniela de França Barros ${ }^{1}$, Marcelo Ferreira Torres ${ }^{2}$ \& Flávia Lucena Frédou ${ }^{3,4}$ \\ ${ }^{1}$ Faculdade de Oceanografia, Universidade Federal do Pará - UFPA, Rua Augusto Corrêa, 01, Guamá, \\ CEP 66075-110,Belém, PA, Brasil,e-mail: dani_fbarros@yahoo.com.br \\ ${ }^{2}$ Instituto Federal de Ensino, Ciência e Tecnologia do Pará, Campus Castanhal, Rod. BR-316, Km 62, \\ Saudade, CEP 68740-970, Castanhal, PA, Brasil,e-mail: marceloferreiratorres@hotmail.com \\ ${ }^{3}$ Departamento de Pesca e Aquicultura, Universidade Federal Rural de Pernambuco - UFRPE, \\ Rua Dom Manoel de Medeiros, Dois Irmãos, s/n, CEP 52171-900, Recife, PE, Brasil \\ ${ }^{4}$ Autor para correspondência: Flávia Lucena Frédou, e-mail: flavia.lucena@pq.cnpq.br
}

BARROS, D.F., TORRES, M.F. \& FRÉDOU, F.L. Ichthyofauna of the estuary of São Caetano de Odivelas and Vigia (Pará, Amazon Estuary). Biota Neotrop. 11(2): http://www.biotaneotropica.org.br/v11n2/en/abstra ct?inventory+bn04111022011

\begin{abstract}
Fish diversity in the Amazon Basin is recognized as one of the highest in the world. However, information on the estuarine ichthyofauna of the region is still fragmented and based on localized inventories. This study reports on an inventory carried out in the estuaries of São Caetano de Odivelas and Vigia, State of Pará, a poorly known area within the Amazon Estuary. Two research cruises of four days long each were undertaken in 2003, one in June (austral winter) and other in December (summer), with use of gill nets, traps, cast net and hook-and-line. It was collected 1,689 individuals, including 58 species from 23 families, all of them previously recorded in the Amazon Estuary. Perciformes, Siluriformes and Clupeiformes were the most diverse taxa, comprising $73.8 \%$ of the species. From the 58 recorded species, $24 \%$ are pelagic, $50 \%$ are demersal and the remaining have pelagic-demersal habits. Coastal-marine species predominated in the region.
\end{abstract}

Keywords: fish, northeast Pará, Amazon.

BARROS, D.F., TORRES, M.F. \& FRÉDOU, F.L. Ictiofauna do estuário de São Caetano de Odivelas e Vigia (Pará, Estuário Amazônico). Biota Neotrop. 11(2): http://www.biotaneotropica.org.br/v11n2/pt/abstract?inve ntory+bn04111022011

Resumo: A diversidade de peixes da Bacia Amazônica é reconhecida como uma das mais altas do mundo. No entanto, o conhecimento acerca da ictiofauna do estuário do Rio Amazonas é fragmentado e baseado em levantamentos localizados. O presente trabalho apresenta um inventário da ictiofauna dos estuários de São Caetano de Odivelas e Vigia, Pará, numa área ainda pouco conhecida do estuário Amazônico. Foram efetuadas duas campanhas de coleta em 2003, com duração de quatro dias cada, uma em junho (inverno) e outra em dezembro (verão), com uso de diferentes artes de pesca (redes de emalhar, currais, tarrafas e linha). Foram coletados 1.689 indivíduos pertencentes a 58 espécies distribuídas em 23 famílias, todas com ocorrência anteriormente registrada no estuário amazônico. As ordens Perciformes, Siluriformes e Clupeiformes foram os grupos mais diversificados, abrangendo 73,8\% das espécies. Das 58 espécies registradas, 24\% são pelágicas, 50\% são demersais e as demais têm hábitos pelágico-demersais. Espécies de hábitos costeiro-marinho predominaram na região.

Palavras-chave: peixes, nordeste paraense, Amazônia. 


\section{Introdução}

Estuários são corpos de água costeira semifechados com ligação livre com o oceano, estendendo-se rio acima até o limite da influência da maré, sendo que em seu interior a água do mar é diluída pela água doce de drenagens continentais (Miranda et al. 2002). Estima-se que $20 \%$ das espécies de peixes marinhos do Brasil utilizem ambientes estuarinos de forma mais ou menos permanente (Haimovici \& Klippel 1999), para crescimento, alimentação e berçário (Viana et al. 2010). Muitos dos peixes encontrados nos estuários são tolerantes a salinidades baixas, enquanto outros adentram os estuários apenas de acordo com a variação da pluma estuarina (Whitfield 1999).

O conhecimento científico sobre a fauna da costa norte do Brasil ainda é escasso, com informações difusas e várias lacunas geográficas. Estudos realizados entre o Amapá e o Maranhão registraram cerca de 303 espécies pertencentes a 23 ordens e 86 famílias, as quais estão distribuídas entre duas subáreas principais: a região das reentrâncias maranhenses-paraenses e a região entre o estuário Amazônico e a costa do Amapá (Camargo \& Isaac 2001). Apesar de inventários de ictiofauna estarem disponíveis para alguns estuários Amazônicos, como os dos rios Curuçá (Giarrizzo \& Krumme 2007) e Caeté (Barletta et al. 2003) e das baías de Marajó (Barthem 1985) e Guajará (Viana et al. 2010), vários complexos estuarinos dessa região ainda permanecem pouco ou totalmente desconhecidos. Neste sentido, o presente trabalho apresenta um inventário ictiofaunístico dos estuários e da região litorânea dos municípios de São Caetano de Odivelas e Vigia, Pará, os quais abrigam espécies de peixes marinhos e de água doce importantes do ponto de vista ecológico e pesqueiro.

\section{Material e Métodos}

A área de estudo foi a região dos municípios de São Caetano de Odivelas e Vigia, no estado do Pará (Figura 1). O clima da região é equatorial amazônico do tipo Am, segundo a classificação de Köppen. A temperatura média anual é de $26^{\circ} \mathrm{C}$ e as precipitações pluviométricas são maiores entre janeiro a junho, diminuindo entre julho e dezembro (Rayol 2002). Os municípios são atravessados por vários rios orientados no sentido Sul-Norte que deságuam diretamente no Oceano Atlântico.

A distribuição das amostragens foi feita de forma a cobrir diferentes ambientes em uma parcela representativa do gradiente de salinidade. Para isso, foi amostrada a região mais interna do estuário, caracterizada por ambientes florestados (mangue-branco e mangue-vermelho) e mais sujeita a oscilações de salinidade, bem como a região caracterizada por exposição maior às correntes e com maior variação do tipo de substrato (fácies lamosas a areno-lamosas). Foram efetuadas duas campanhas de amostragem em 2003, com duração de quatro dias cada, a primeira no final da estação chuvosa, denominada inverno (junho), com oito amostras (cada lance de pesca representou uma amostra) e a segunda no final da estação seca, denominada verão (dezembro), na qual foram realizadas nove amostras (Figura 1).

Para a realização das amostragens foi utilizada uma embarcação local empregada na pesca artesanal, com emprego de várias artes de pesca. Duas redes de espera com malha de $30 \mathrm{~mm}$ e $40 \mathrm{~mm}$ entre nós opostos, ambas com $25 \mathrm{~m}$ de comprimento e $2 \mathrm{~m}$ de altura, foram colocadas à deriva por entre 100 e 120 minutos. Uma rede de espera de malha $30 \mathrm{~mm}$ entre nós foi utilizada para a tapagem de igarapé, tendo permanecido submersa e fixada com estacas de madeiras durante um ciclo de maré. Este mesmo procedimento foi empregado com um puçá de camarão com tamanho de malha de $7 \mathrm{~mm}$ entre nós, $3 \mathrm{~m}$ de comprimento e $2 \mathrm{~m}$ de altura. Nas áreas mais abertas, em ambientes praiais de substrato arenoso, a ictiofauna capturada em currais de pescadores artesanais também foi amostrada. Uma rede de arrasto de praia com $12 \times 1,5 \mathrm{~m}$ e malha de $10 \mathrm{~mm}$ entre nós foi utilizada em um ambiente de mangue. Pescarias com linha de mão também foram empregadas, utilizando-se anzóis de números 12 , 15 e 19 (anzóis de pequeno comprimento total). Uma tarrafa com 3,0 $\mathrm{m}$ de diâmetro e malha $25 \mathrm{~mm}$ entre nós foi empregada para a captura de cardumes de engraulídeos em águas abertas. As redes de emalhar, currais, tarrafa e linhas de mão foram utilizadas em ambos os períodos de coletas. Amostras com puçá de camarão e tapagem de igarapé foram efetuadas somente no verão, enquanto que a rede de arrasto de praia foi utilizada apenas no inverno.

A curva de acumulação de espécies (Magurran 2004) não atingiu uma assíntota, indicando que a amostragem não foi exaustiva. Assim, a riqueza de espécies foi estimada com um método não paramétrico de estimativa por extrapolação (Chao 1984), selecionado dada à sua adequabilidade nas situações de pequenas amostras e disponibilidade de dados quantitativos (Colwell \& Coddington 1994, Magurran 2004). O índice e os desvios padrão das estimativas foram calculados utilizando a equação analítica proposta por Colwell et al. (2004), através do programa EstimateS v.8 (Colwell 2009).

Após as capturas, os peixes foram triados a bordo e preservados em gelo ou formol a $10 \%$. O material foi identificado em nível de espécie através chaves dicotômicas (Cervigón 1991, 1993, 1994, 1996, FAO 1992, Cervigón et al. 1992), estando listado de acordo com Nelson (1994) e Froese \& Pauly (2007). Exemplares-testemunho preservados em álcool $70 \%$ estão depositados no Laboratório de Dinâmica, Avaliação e Manejo de Recursos Pesqueiros (DIAMAR) da Universidade Federal do Pará. Informações quanto à tolerância à variação de salinidade e hábitos de cada espécie foram obtidas em Camargo \& Isaac (2001), Krumme et al. (2004) e Froese \& Pauly (2007).

\section{Resultados}

Um total de 1.689 indivíduos foi coletado, resultando no registro de 58 espécies distribuídas em 23 famílias. A riqueza estimada por extrapolação (64 espécies) foi superior quando comparada à riqueza observada (Sobs) (Figura 2), embora a estimativa sugira que uma grande parcela das espécies tenha sido efetivamente amostrada através das coletas $(91 \%)$.

As ordens Perciformes (25 espécies), Siluriformes (dez espécies) e Clupeiformes (dez espécies) foram os grupos com maior riqueza, compreendendo $73,8 \%$ das espécies (Tabela 1). Deste total, cerca de $36 \%$ das espécies têm importância comercial no estado do Pará. As quatro espécies mais abundantes, em número de indivíduos, corresponderam a $65 \%$ do total de indivíduos capturados: Cetengraulis edentulus (Cuvier, 1829), Mugil gaimardianus Desmarest, 1831, Macrodon ancylodon (Bloch \& Schneider 1801) e Colomesus psittacus (Bloch \& Schneider 1801).Verificou-se que $33 \%$ das espécies capturadas ocorreram em ambos períodos, enquanto $34 \%$ ocorreram exclusivamente no verão e $31 \%$ apenas no inverno (Tabela 1).

Das 58 espécies registradas, $24 \%$ são pelágicas, $50 \%$ são demersais e as demais têm hábitos pelágico-demersais (Tabela 1). Dentre 53 espécies cuja tolerância das variações de salinidade foi determinada, $14 \%$ são de origem límnica, enquanto $62 \%$ são costeiro-marinhas. As demais espécies são verdadeiramente eurialinas, com ampla distribuição nos estuários (Tabela 1).

\section{Discussão}

Em estuários, onde as diferenças nos fatores ambientais são agudas, as alterações nas propriedades físicas, químicas e biológicas resultam num pronunciado estresse ambiental que pode estar relacionado com sua baixa diversidade quando comparados com 
outros sistemas aquáticos menos variantes (Kennish 1986). Um total de 58 espécies foi registrado na área de estudo, com predominância das ordens Perciformes, Siluriformes e Clupeiformes. Considerando outros levantamentos de ictiofauna estuarina no Pará, essa riqueza de espécies é similar àquela conhecida nos estuários do Rio Curuçá [65 espécies, Giarrizzo \& Krumme (2007)] e Rio Caeté [49 espécies, Barletta et al. (2003)], sendo superior ao número de espécies registradas no estuário da Baía de Guajará [40 espécies, Viana et al. (2010)]. As diferenças observadas nos diversos levantamentos ictiofaunísticos do estuário Amazônico é um reflexo da variabilidade nas propriedades físico-químicas da água, hidrodinâmica e características do sedimento, entre outros fatores. Adicionalmente, essa heterogeneidade pode também estar relacionada com artifícios dos métodos e do esforço amostral dissimilar.

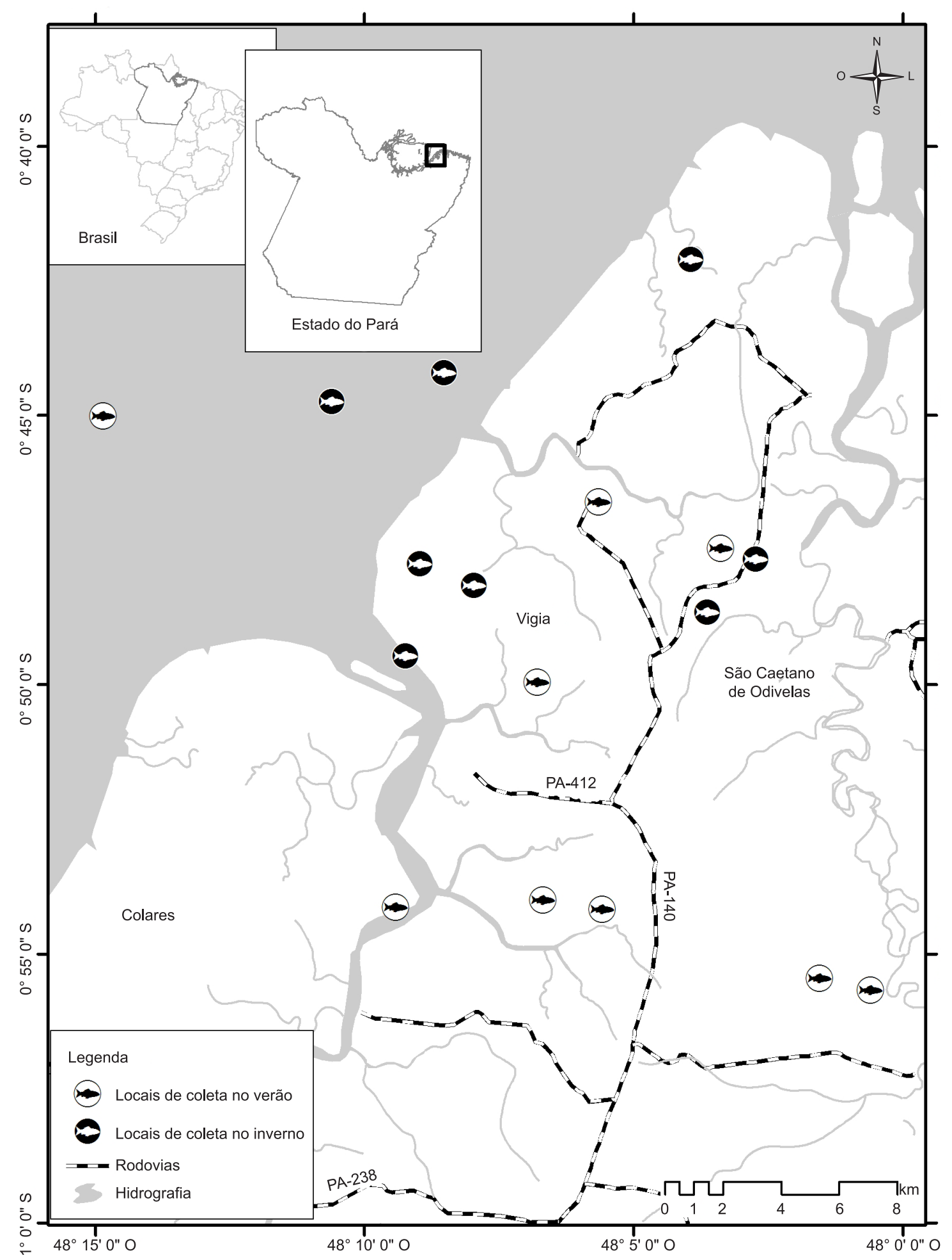

Figura 1. Área de estudo na $1^{\mathrm{a}}$ (inverno) e $2^{\mathrm{a}}$ campanhas (verão). Mapa elaborado a partir da base de dados da CPRM (CPRM, 2008).

Figure 1. Study area in the $1^{\text {st }}$ (winter) and $2^{\text {nd }}$ cruises (summer). Map created from the CPRM (CPRM, 2008) database. 


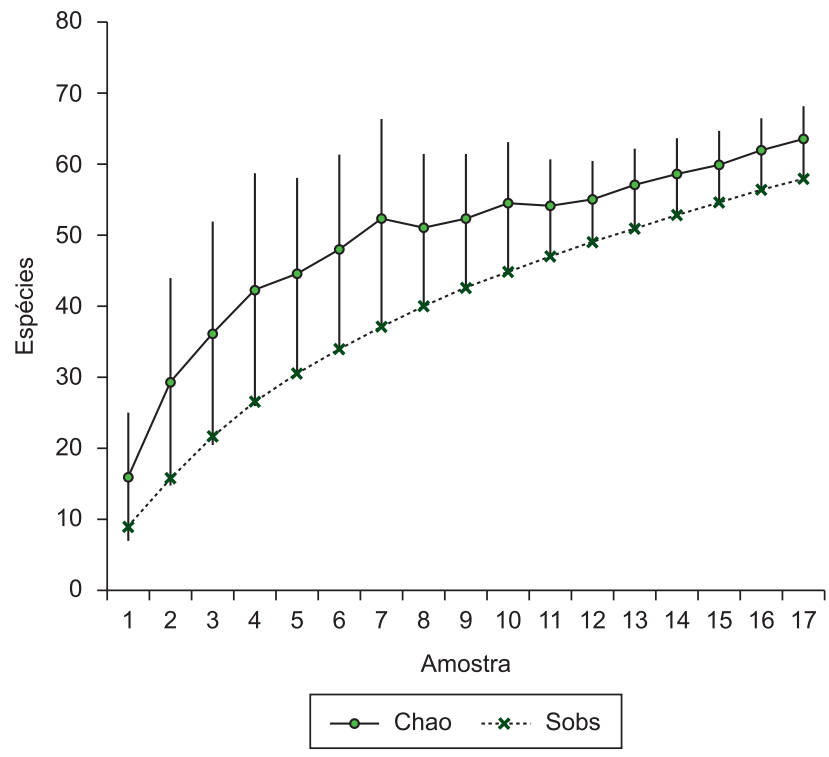

Figura 2. Curva de acumulação de espécies do estuário de São Caetano de Odivelas e Vigia (Sobs) e curva de estimativa de riqueza por extrapolação (Chao). Barras representam o desvio padrão das estimativas Chao.

Figure 2. Species accumulation curve of the estuary of São Caetano de Odivelas and Vigia (Sobs) and species richness estimator (Chao). Bars represent the standard deviation of estimates Chao.
A região estuarina amazônica apresenta uma hidrodinâmica considerável, com grandes variações espaciais e temporais em sua estrutura hidrográfica (Geyer at al. 1991). Estuários com maior estabilidade hídrica e elevadas salinidades são mais favoráveis à exploração por um maior número de espécies de peixes (Nicolas et al. 2010, Thiel et al. 2003). A baía de Guajará, por exemplo, com elevada hidrodinâmica e baixa salinidade $(<1)$, apresenta menor riqueza (Viana et al. 2010) quando comparada com outros estuários Amazônicos (Barletta et al. 2003, Krumme et al. 2004, Giarrizo \& Krumme 2007). Essa baixa riqueza de espécies também pode também estar relacionada com a composição e instabilidade do sedimento (Elliott \& McLusky 2002).

São poucas as espécies que passam todo seu ciclo de vida em ambientes estuarinos (Camargo \& Isaac 2001). A maior parte das espécies de peixes encontradas em estuários é visitante e utiliza esse ambiente apenas em determinados momentos, especialmente como área de reprodução, berçário e alimentação. Os estuários apresentam elevada disponibilidade de alimento e refúgio, em função de sua complexidade estrutural, baixas profundidades, elevada turbidez e alta produtividade (Knox 1986, Kennish 1986, Valesini et al. 1997, Spach et al. 2003, Harrison \& Whitfield 2004, Mafalda Junior et al. 2004). Nos estuários onde há maior influência da água salgada e os valores médios de salinidade são mais elevados, o número de espécies dulcícolas que suportam águas salobras e que entram nos estuários apenas eventualmente é menor, como observados nos estuário dos rios Curuçá e Caeté, onde a salinidade variou entre 6-41 e 6-35,

Tabela 1. Lista de espécies, número de indivíduos $(\mathrm{N})$ e distribuição em relação a estação ( $\mathrm{I}=$ Inverno e $\mathrm{V}=\mathrm{Verão})$, ambiente preferencial $(\mathrm{L}=\mathrm{Límnico}$; $\mathrm{E}=$ Estuarino; $\mathrm{C}=$ Costeiro/Marinho) e hábitat ( $\mathrm{D}=$ Demersal; $\mathrm{P}=$ Pelágico). * Salinidade média: 12,9. ** Salinidade média: 21.

Table 1. List of species, number of individuals $(\mathrm{N})$ and distribution in relation to season ( $\mathrm{I}$ e V = winter and summer), preferred environmental ( $\mathrm{L}=\mathrm{Límnico}$; $\mathrm{E}=$ Estuarine; $\mathrm{C}=\mathrm{Coastal} /$ Marine $)$ and habitat $(\mathrm{D}=$ Demersal; $\mathrm{P}=$ Pelagic). * Mean salinity: 12.9. ** Mean salinity: 21.

\begin{tabular}{|c|c|c|c|c|c|c|c|c|}
\hline \multirow[t]{2}{*}{ Ordem/Família/Espécie } & \multirow[t]{2}{*}{$\operatorname{Fr}(\mathbf{N})$} & \multicolumn{2}{|l|}{ Estação } & \multicolumn{2}{|c|}{$\begin{array}{c}\text { Tipo de } \\
\text { ambiente } \\
\text { preferencial }\end{array}$} & \multicolumn{3}{|c|}{ Habitat } \\
\hline & & $I^{*}$ & $\mathbf{V}^{* *}$ & $\mathbf{L}$ & $\mathbf{E}$ & $\mathbf{C}$ & D & $\mathbf{P}$ \\
\hline \multicolumn{9}{|l|}{ CLUPEIFORMES } \\
\hline \multicolumn{9}{|l|}{ Engraulidae } \\
\hline Anchovia clupeoides (Swainson, 1839) & 3 & - & $\mathrm{X}$ & - & $\mathrm{X}$ & $\mathrm{X}$ & $X$ & $\mathrm{X}$ \\
\hline Anchoa hepsetus (Linnaeus, 1758) & 5 & - & $\mathrm{X}$ & $\mathrm{X}$ & $\mathrm{X}$ & $\mathrm{X}$ & - & $\mathrm{X}$ \\
\hline Cetengraulis edentulus (Cuvier, 1829) & 715 & $\mathrm{X}$ & $\mathrm{X}$ & - & $\mathrm{X}$ & $X$ & - & $\mathrm{X}$ \\
\hline Lycengraulis batesii (Günther, 1868) & 12 & $\mathrm{X}$ & - & $\mathrm{X}$ & $\mathrm{X}$ & - & - & $\mathrm{X}$ \\
\hline Lycengraulis grossidens Agassiz, 1829 & 63 & - & $\mathrm{X}$ & $\mathrm{X}$ & $\mathrm{X}$ & $\mathrm{X}$ & - & $\mathrm{X}$ \\
\hline Pterengraulis atherinoides Linnaeus, 1766 & 2 & - & $X$ & $\mathrm{X}$ & $\mathrm{X}$ & - & - & $\mathrm{X}$ \\
\hline \multicolumn{9}{|l|}{ Clupeidae } \\
\hline Opisthonema oglinum Lesueur, 1818 & 1 & - & $\mathrm{X}$ & - & $\mathrm{X}$ & $\mathrm{X}$ & - & $\mathrm{X}$ \\
\hline Pellona sp. & 2 & - & $\mathrm{X}$ & - & $\mathrm{X}$ & - & - & \\
\hline Sardinella janeiro (Eigenmann, 1894) & 7 & $\mathrm{X}$ & - & - & $\mathrm{X}$ & $\mathrm{X}$ & - & $\mathrm{X}$ \\
\hline \multicolumn{9}{|l|}{ Pristigasteridae } \\
\hline Odontognathus mucronatus Lacepéde, 1800 & 2 & - & $\mathrm{X}$ & $\mathrm{X}$ & $\mathrm{X}$ & $\mathrm{X}$ & - & $\mathrm{X}$ \\
\hline \multicolumn{9}{|l|}{ SILURIFORMES } \\
\hline \multicolumn{9}{|l|}{ Ariidae } \\
\hline Aspistor quadriscutis (Valenciennes, 1840) & 6 & $\mathrm{X}$ & - & - & $\mathrm{X}$ & $\mathrm{X}$ & $\mathrm{X}$ & $\mathrm{X}$ \\
\hline Amphiarius phrygiatus (Valenciennes, 1840) & 3 & $\mathrm{X}$ & - & - & $\mathrm{X}$ & $\mathrm{X}$ & $\mathrm{X}$ & - \\
\hline Sciades couma (Valenciennes, 1840) & 39 & $\mathrm{X}$ & $\mathrm{X}$ & $\mathrm{X}$ & $\mathrm{X}$ & $\mathrm{X}$ & $\mathrm{X}$ & - \\
\hline Sciades proops (Valenciennes, 1840) & 3 & - & $\mathrm{X}$ & - & $\mathrm{X}$ & $\mathrm{X}$ & $\mathrm{X}$ & - \\
\hline Arius sp. & 1 & $\mathrm{X}$ & - & - & $\mathrm{X}$ & - & - & - \\
\hline Bagre bagre (Linnaeus, 1766) & 24 & $\mathrm{X}$ & $\mathrm{X}$ & - & $\mathrm{X}$ & $\mathrm{X}$ & $\mathrm{X}$ & - \\
\hline
\end{tabular}




\begin{tabular}{|c|c|c|c|c|c|c|c|c|}
\hline \multirow[t]{2}{*}{ Ordem/Família/Espécie } & \multirow[t]{2}{*}{$\operatorname{Fr}(\mathbf{N})$} & \multicolumn{2}{|l|}{ Estação } & \multicolumn{2}{|c|}{$\begin{array}{c}\text { Tipo de } \\
\text { ambiente } \\
\text { preferencial }\end{array}$} & \multicolumn{3}{|c|}{ Habitat } \\
\hline & & $I^{*}$ & $\mathbf{V}^{* * *}$ & $\mathbf{L}$ & $\mathbf{E}$ & $\mathbf{C}$ & D & $\mathbf{P}$ \\
\hline Cathorops spixii (Agassiz, 1829) & 28 & $\mathrm{X}$ & $\mathrm{X}$ & - & $\mathrm{X}$ & $\mathrm{X}$ & $\mathrm{X}$ & - \\
\hline \multicolumn{9}{|l|}{ Auchenipteridae } \\
\hline Pseudauchenipterus nodosus (Bloch, 1794) & 22 & - & $\mathrm{X}$ & $\mathrm{X}$ & $\mathrm{X}$ & - & $\mathrm{X}$ & - \\
\hline \multicolumn{9}{|l|}{ BATRACHOIDIFORMES } \\
\hline \multicolumn{9}{|l|}{ Batrachoididae } \\
\hline $\begin{array}{l}\text { Batrachoides surinamensis Bloch \& Schneider, } \\
1801\end{array}$ & 12 & $\mathrm{X}$ & $\mathrm{X}$ & - & $X$ & $\mathrm{X}$ & $\mathrm{X}$ & - \\
\hline Talassophryne maculosa Günther, 1861 & 2 & $\mathrm{X}$ & $\mathrm{X}$ & - & $\mathrm{X}$ & $\mathrm{X}$ & $\mathrm{X}$ & - \\
\hline \multicolumn{9}{|l|}{ MUGILIFORMES } \\
\hline \multicolumn{9}{|l|}{ Mugilidae } \\
\hline Mugil cephalus Linnaeus, 1758 & 9 & $\mathrm{x}$ & - & $\mathrm{X}$ & $\mathrm{X}$ & $\mathrm{X}$ & $\mathrm{X}$ & $\mathrm{X}$ \\
\hline Mugil gaimardianus Desmarest, 1831 & 161 & $\mathrm{X}$ & - & $\mathrm{X}$ & $\mathrm{X}$ & - & - & - \\
\hline Mugil incilis Hancock, 1830 & 16 & $\mathrm{X}$ & - & - & $\mathrm{X}$ & $\mathrm{X}$ & $\mathrm{X}$ & - \\
\hline Mugil sp.1 & 91 & $\mathrm{X}$ & $\mathrm{X}$ & - & $\mathrm{X}$ & - & - & - \\
\hline \multicolumn{9}{|l|}{ BELONIFORMES } \\
\hline \multicolumn{9}{|l|}{ Belonidae } \\
\hline Strongylura timucu (Walbaum, 1792) & 4 & $\mathrm{x}$ & - & $\mathrm{X}$ & $\mathrm{X}$ & $\mathrm{X}$ & - & $\mathrm{X}$ \\
\hline \multicolumn{9}{|l|}{ Hemirhanphidae } \\
\hline Hemiramphus brasiliensis (Linnaeus, 1758) & 2 & - & $\mathrm{X}$ & - & $\mathrm{X}$ & $\mathrm{X}$ & $\mathrm{X}$ & - \\
\hline \multicolumn{9}{|l|}{ CYPRINODONTIFORMES } \\
\hline \multicolumn{9}{|l|}{ Anablepidae } \\
\hline Anableps anableps (Linnaeus, 1758) & 17 & $\mathrm{X}$ & $\mathrm{X}$ & $\mathrm{X}$ & $\mathrm{X}$ & - & $\mathrm{X}$ & - \\
\hline \multicolumn{9}{|l|}{ PERCIFORMES } \\
\hline \multicolumn{9}{|l|}{ Echeneidae (Echeneididae) } \\
\hline Echeneis naucrates Linnaeus, 1758 & 1 & $\mathrm{X}$ & - & - & $\mathrm{X}$ & $\mathrm{X}$ & - & - \\
\hline \multicolumn{9}{|l|}{ Carangidae } \\
\hline Caranx hippos (Linnaeus, 1766) & 1 & $\mathrm{X}$ & - & $\mathrm{X}$ & $\mathrm{X}$ & $\mathrm{x}$ & - & $\mathrm{X}$ \\
\hline Chloroscombrus chrysurus (Linnaeus, 1766) & 34 & - & $\mathrm{X}$ & - & $\mathrm{X}$ & $\mathrm{X}$ & - & $\mathrm{X}$ \\
\hline Oligoplites palometa (Cuvier, 1832) & 22 & $\mathrm{X}$ & $\mathrm{X}$ & $\mathrm{X}$ & $\mathrm{X}$ & $\mathrm{X}$ & - & $\mathrm{X}$ \\
\hline Selene vomer (Linnaeus, 1758) & 5 & $\mathrm{X}$ & $\mathrm{X}$ & - & $\mathrm{X}$ & $\mathrm{x}$ & $\mathrm{X}$ & - \\
\hline Trachinotus cayennensis Cuvier, 1832 & 12 & $\mathrm{X}$ & - & - & $\mathrm{X}$ & $\mathrm{x}$ & $\mathrm{X}$ & - \\
\hline Trachinotus carolinus (Linnaeus, 1766) & 2 & - & $\mathrm{X}$ & - & $\mathrm{X}$ & $\mathrm{x}$ & $\mathrm{X}$ & $\mathrm{X}$ \\
\hline \multicolumn{9}{|l|}{ Gerreidae } \\
\hline Gerres cinereus (Walbaum, 1792) & 1 & - & $\mathrm{X}$ & - & $\mathrm{X}$ & $\mathrm{x}$ & - & $\mathrm{X}$ \\
\hline \multicolumn{9}{|l|}{ Haemulidae (Pomadasyidae) } \\
\hline Conodon nobilis (Linnaeus, 1758) & 4 & - & $\mathrm{X}$ & - & $\mathrm{X}$ & $\mathrm{x}$ & $\mathrm{x}$ & - \\
\hline Genyatremus luteus (Bloch, 1795) & 14 & $\mathrm{X}$ & $\mathrm{X}$ & - & $\mathrm{X}$ & $\mathrm{x}$ & $\mathrm{X}$ & - \\
\hline Sciaenidae & & & & & & & & \\
\hline Cynoscion acoupa (Lacepède, 1801) & 26 & $\mathrm{X}$ & $\mathrm{X}$ & $\mathrm{X}$ & $\mathrm{X}$ & $\mathrm{x}$ & $\mathrm{X}$ & - \\
\hline Cynoscion leiarchus (Cuvier, 1830) & 9 & $\mathrm{x}$ & $\mathrm{X}$ & - & $\mathrm{X}$ & $\mathrm{x}$ & $\mathrm{x}$ & - \\
\hline Cynoscion microlepidotus (Cuvier, 1830) & 6 & $\mathrm{X}$ & - & - & $\mathrm{X}$ & $\mathrm{x}$ & $\mathrm{X}$ & - \\
\hline $\begin{array}{l}\text { Macrodon ancylodon (Bloch \& Schneider, } \\
\text { 1801) }\end{array}$ & 111 & $\mathrm{X}$ & $\mathrm{X}$ & - & $\mathrm{X}$ & $\mathrm{x}$ & $\mathrm{X}$ & - \\
\hline Menticirrhus americanus (Linnaeus, 1758) & 2 & - & $\mathrm{X}$ & $\mathrm{X}$ & $\mathrm{X}$ & $\mathrm{x}$ & $\mathrm{X}$ & - \\
\hline Micropogonias furnieri (Desmarest, 1823) & 12 & $\mathrm{x}$ & $\mathrm{X}$ & $\mathrm{X}$ & $\mathrm{X}$ & $\mathrm{x}$ & $\mathrm{X}$ & - \\
\hline Plagioscion auratus (Castelnau, 1855) & 2 & - & $\mathrm{X}$ & $\mathrm{X}$ & $\mathrm{X}$ & - & $\mathrm{X}$ & $\mathrm{X}$ \\
\hline Stellifer microps (Steindachner, 1864) & 1 & - & $\mathrm{X}$ & $\mathrm{X}$ & $\mathrm{X}$ & $\mathrm{X}$ & $\mathrm{X}$ & - \\
\hline Stellifer rastrifer (Jordan, 1889) & 10 & - & $\mathrm{X}$ & $\mathrm{X}$ & $\mathrm{X}$ & $\mathrm{x}$ & $\mathrm{X}$ & - \\
\hline Stellifer $\mathrm{sp} .1$ & 4 & - & $\mathrm{X}$ & - & $\mathrm{X}$ & - & - & - \\
\hline
\end{tabular}


Tabela 1. Continuação...

\begin{tabular}{|c|c|c|c|c|c|c|c|c|}
\hline \multirow[t]{2}{*}{ Ordem/Família/Espécie } & \multirow[t]{2}{*}{$\operatorname{Fr}(\mathbf{N})$} & \multicolumn{2}{|l|}{ Estação } & \multicolumn{2}{|c|}{$\begin{array}{c}\text { Tipo de } \\
\text { ambiente } \\
\text { preferencial }\end{array}$} & \multicolumn{3}{|c|}{ Habitat } \\
\hline & & $\mathbf{I}^{*}$ & $\mathbf{V} * *$ & $\mathbf{L}$ & $\mathbf{E}$ & $\mathbf{C}$ & D & $\mathbf{P}$ \\
\hline Stellifer sp.2 & 1 & - & $\mathrm{X}$ & - & $\mathrm{X}$ & - & - & - \\
\hline \multicolumn{9}{|l|}{ Stromateidae } \\
\hline Peprilus paru (Linnaeus, 1758) & 8 & $\mathrm{X}$ & $\mathrm{X}$ & - & $X$ & $\mathrm{X}$ & $\mathrm{X}$ & $\mathrm{X}$ \\
\hline \multicolumn{9}{|l|}{ Gobiidae } \\
\hline Gobioides broussonneti Lacepéde, 1800 & 1 & $X$ & - & $\mathrm{X}$ & $X$ & $\mathrm{X}$ & $\mathrm{X}$ & - \\
\hline \multicolumn{9}{|l|}{ Trichiuridae } \\
\hline Trichiurus lepturus Linnaeus, 1758 & 2 & $\mathrm{X}$ & - & - & $X$ & $X$ & $\mathrm{X}$ & $\mathrm{X}$ \\
\hline \multicolumn{9}{|l|}{ Scombridae } \\
\hline $\begin{array}{l}\text { Scomberomorus brasiliensis Collete, Russo \& } \\
\text { Zavala-Camin, } 1978\end{array}$ & 19 & $\mathrm{X}$ & - & - & $\mathrm{X}$ & $\mathrm{X}$ & - & $\mathrm{X}$ \\
\hline \multicolumn{9}{|l|}{ PLEURONECTIFORMES } \\
\hline \multicolumn{9}{|l|}{ Achiridae } \\
\hline Achirus achirus (Linnaeus, 1758) & 4 & $\mathrm{X}$ & $\mathrm{X}$ & $\mathrm{X}$ & $\mathrm{X}$ & $\mathrm{X}$ & $\mathrm{X}$ & - \\
\hline Achirus lineatus (Linnaeus, 1758) & 1 & $\mathrm{X}$ & - & - & $\mathrm{X}$ & $\mathrm{X}$ & $\mathrm{X}$ & - \\
\hline \multicolumn{9}{|l|}{ Bothidae } \\
\hline Citharichthys spilopterus Gunther, 1862 & 3 & $X$ & $\mathrm{X}$ & $\mathrm{X}$ & $X$ & $\mathrm{X}$ & $\mathrm{X}$ & - \\
\hline \multicolumn{9}{|l|}{ Cynoglossidae } \\
\hline Symphurus plagusia (Bloch \& Schneider, 1801) & 1 & $\mathrm{X}$ & - & - & $X$ & $\mathrm{X}$ & $\mathrm{X}$ & - \\
\hline \multicolumn{9}{|l|}{ TETRAODONTIFORMES } \\
\hline \multicolumn{9}{|l|}{ Tetraodontidae } \\
\hline Colomesus psittacus (Bloch \& Schneider, 1801) & 103 & $\mathrm{X}$ & $\mathrm{X}$ & $\mathrm{X}$ & $\mathrm{X}$ & $\mathrm{X}$ & $\mathrm{X}$ & - \\
\hline Sphoeroides testudineus Linnaeus, 1758 & 15 & $\mathrm{X}$ & $\mathrm{X}$ & - & $\mathrm{X}$ & $\mathrm{X}$ & $\mathrm{X}$ & - \\
\hline
\end{tabular}

respectivamente (Barletta et al. 2003, Giarrizzo \& Krumme 2007). No presente estudo também foi registrada uma riqueza de espécies alta, com predomínio de espécies costeiras/marinhas em águas de salinidade relativamente elevada (média de 17). Adicionalmente, dado o predomínio de espécies demersais em águas pouco profundas da plataforma continental do estuário amazônico, onde a cadeia detritívora apresenta um grande número de espécies e elevada biomassa (Torres 1999), se destacaram neste estudo, espécies de hábito demersal.

Este trabalho supre uma lacuna geográfica importante no conhecimento da ictiofauna estuarina Amazônica. O inventariamento das espécies e o reconhecimento dos principais fatores que influenciam sua distribuição é uma etapa fundamental para o conhecimento da estrutura e do funcionamento dinâmico desses sistemas, dos quais as populações locais dependem fortemente como fonte de proteína e renda (Barletta \& Costa 2009, Costa et al. 2009).

\section{Agradecimentos}

Agradecemos ao Programa Integrado de Apoio ao Ensino, Pesquisa e Extensão (PROINT-UFPA), ao Programa Institucional de Bolsas de Iniciação Científica (PIBIC-UFPA) e ao Conselho Nacional de Desenvolvimento Científico e Tecnológico pela bolsa de pesquisa ao terceiro autor (Proc. 302280/2007-3). Ronaldo Santos colaborou na triagem e identificação das espécies.

\section{Referências Bibliográficas}

BARLETTA, M. \& COSTA, M.F. 2009. Living and nonliving resources exploitation in a tropical semi-arid estuary. J. Coast. Res. 56:371-375.
BARLETTA, M., BARLETTA-BERGAN, A., SAINT-PAUL, U. \& HUBOLD, G. 2003. Seasonal changes in density, biomass, and diversity of estuarine fishes in tidal mangrove creeks of the lower Caeté Estuary (northern Brazilian coast, east Amazon). Mar. Ecol. Prog. Ser. 256:217228. http://dx.doi.org/10.3354/meps256217

BARTHEM, R.B. 1985. Ocorrência, distribuição e biologia dos peixes da baía de Marajó, estuário amazônico. Bol. Mus. Para. Emílio Goeldi, ser. Zool. 2(1):49-69.

CAMARGO, M. \& ISAAC, V. 2001 Os peixes estuarinos da região norte do Brasil: lista de espécies e considerações sobre sua distribuição geográfica. Bol. Mus. Para. Emílio Goeldi, ser. Zool. 17(2):133-157.

CERVIGÓN, F. 1991. Los peces marinos de Venezuela. Fundación cientifica Los Roques, Caracas, v, 1.

CERVIGÓN, F. 1993. Los peces marinos de Venezuela. Fundación cientifica Los Roques, Caracas, v.2.

CERVIGÓN, F. 1994. Los peces marinos de Venezuela. Fundación cientifica Los Roques, Caracas, v.3.

CERVIGÓN, F. 1996. Los peces marinos de Venezuela. Fundación cientifica Los Roques, Caracas, v.4.

CERVIGÓN, F., CIPRIANI, R., FISCHER, W., GARIBALDI, L., HENDRICKX, M., LEMUS, A.J., MÁRQUEZ, R., POUTIERS, J.M., ROBIANA, G. \& RODRIGUEZ, B. 1992. Fichas FAO de identification de especies para los fines de la pesca: Especies comerciales marinas y de aguas salobres de la costa septentrional de sur America. FAO, Roma.

CHAO, A. 1984. Non-parametric estimation of the number of classes in a population. Scand. J. Statist. 11:265-270.

COLWELL, R.K. \& CODDINGTON, J.A. 1994. Estimating terrestrial biodiversity through extrapolation. In Biodiversity, measurement and estimation (D.L. Hawksworth, ed.). Chapman and Hall, New York, p.101-118. http://dx.doi.org/10.1890/03-0557 
COLWELL, R.K., MAO, C.X. \& CHANG, J. 2004. Interpolating, extrapolating, and comparing incidence-based species accumulation curves. Ecology. 85:2717-2727.

COLWELL, R.K. 2009. EstimateS: statistical estimation of species richness and shared species from samples. v. 8.2. http://viceroy.eeb.uconn.edu/ EstimateS. http://purl.oclc.org/estimates (último acesso em 31/05/2011).

COMPANHIA DE PESQUISA DE RECURSOS MINERAIS - CPRM. 2008 Geologia e recursos minerais do estado do Pará: Sistema de informações geográficas - SIG: texto explicativo dos mapas geológicos e tectônicos e de recursos minerais do estado do Pará. Escala 1:1.000.000. CPRM, Belém.

COSTA, M.F., BARBOSA, S.C.T., BARLETTA, M., DANTAS, D.V., KEHRIG, H.A., SEIXAS, T.G. \& MALM, O. 2009. Differences in Mercury accumulation in Trichiurus lepturus (cutlassfish) in relation to length, weight and season. Environ. Sci. Pollut. R. 16:423-430. PMid:19290559. http://dx.doi.org/10.1007/s11356-009-0120-x

ELLIOTT, M. \& McLUSKY, D.S. 2002. The need for definitions in understanding Estuaries. Estuar. Coast. Shelf Sci. 55:815-827. http://dx.doi.org/10.1006/ecss.2002.1031

FOOD AND AGRICULTURE ORGANIZATION - FAO. 1992. Guia de campo de las especies comerciales marinas y de aguas salobres de la costa septentrional del Sur America. NORAD, Roma.

FROESE, R. \& PAULY, D. 2007. FishBase. http://www.fishbase.org

GEYER, W.R., BEARDSLEY, R.C., CANDELA, J., CASTRO, B.M., LEGECKIS, R.V., LENTZ, S.J., LIMEBURNER, R., MIRANDA, L.B. \& TROWBRIDGE, J.H. 1991. The physical oceanography of the Amazon outflow. Oceanography. 4:8-15.

GIARRIZZO, T. \& KRUMME, U. 2007 Spatial differences and seasonal cyclicity in the intertidal fish fauna from four mangrove creeks in a salinity zone of the Curuçá Estuary, North Brazil. Bull. Mar. Sci. 80:739-754.

HAIMOVICI, M. \& KLIPPEL, S. 1999. Diagnóstico da Biodiversidade dos Peixes Teleósteos Demersais Marinhos e Estuarinos do Brasil. PROBIO/Fundação Universidade Federal de Rio Grande, Rio Grande.

HARRISON, T.D. \& WHITFIELD, A.K. 2004. A multi-metric fish index to assess the environmental condition of estuaries. J. Fish Biol. 65:683-710. http://dx.doi.org/10.1111/j.0022-1112.2004.00477.x

KENNISH, M.J. 1986. Ecology of estuaries. CRC PRESS, Flórida, v. 2: Biological Aspects.

KNOX, G.A. 1986. Estuarine Ecosystems: a Systems Approach. CRC Press, Florida.

KRUMME, U., SAINT-PAUL, U. \& ROSENTHAL, H. 2004. Tidal and diel changes in the structure of a nekton assemblage in small intertidal mangrove creeks in northern Brazil. Aquat. Living Resourc. 17:215-229. http://dx.doi.org/10.1051/alr:2004019
MAFALDA JUNIOR, P.O., SINQUE, C., MUELBERT, J.H. \& SOUZA, C.S. 2004. Distribuição e abundância do Ictioplâncton na costa Norte da Bahia, Brasil. Trop. Ocean. 32(1):69-88.

MAGURRAN, A. 2004. Measuring biological diversity. Blackwell Publishing, Oxford.

MIRANDA, L.B., CASTRO, B.M. \& BJÖRN, K. 2002 Princípios de oceanografia física de estuários. Ed. Universidade de São Paulo, São Paulo.

NELSON, J.S. 1994. Fishes of the world. John Wiley \& Sons, Canada.

NICOLAS, D., LOBRY, J., LEPAPE, O. \& BOËT, P. 2010. Functional diversity in European estuaries: Relating the composition of fish assemblages to the abiotic environment. Estuar. Coast. Shelf Sci. 88:329-338. http://dx.doi.org/10.1016/j.ecss.2010.04.010

RAYOL, A.C.D. 2002. Estudo geoeconômico e ambiental dos recursos minerais dos municípios da Vigia, São Caetano de Odivelas e Colares. Trabalho de Conclusão de Curso, Universidade Federal do Pará, Belém.

SPACH, H. L., SANTOS, C. \& GODEFROID, R.S. 2003. Padrões temporais nas assembléias de peixes na Gamboa do Sucuriú, Baía de Paranaguá, Brasil. Rev. Brasil. Zool. 20(4):591-600.

THIEL, R., CABRAL, H. \& COSTA, M.J., 2003. Composition, temporal changes and ecological guild classification of the ichthyofaunas of large European estuaries-a comparison between the Tagus (Portugal) and the Elbe (Germany). J. Appl. Ichthyol. 19:330-342. http://dx.doi. org/10.1046/j.1439-0426.2003.00474.x

TORRES, M.F. 1999. Variação sazonal e espacial da estrutura de comunidades dos peixes demersais da região de foz dos rios Amazonas e Tocantins - PA $\left(0^{\circ} 10^{\prime} \mathrm{S}-2^{\circ} 30^{\prime} \mathrm{N} ; 47^{\circ} 50^{\prime} \mathrm{O}-50^{\circ} 30^{\prime} \mathrm{O}\right)$ - Brasil. Dissertação de Mestrado, Universidade Federal do Pará, Belém.

VALESINI, F.J., POTTER, I.C., PLATELL, M.E. \& HYNDES, G.A. 1997. Ichthyofauna of a temperate estuary and adjacent marine embayment. Implications regarding choice of nursery area and influence of environmental changes. Mar. Biol. 128:317-328. http://dx.doi. org/10.1007/s002270050097

VIANA, A.P., LUCENA FRÉDOU, F., FRÉDOU, T., TORRES, M.F. \& BORDALO, A.O. 2010. Fish fauna as an indicator of environmental quality in an urbanised region of the Amazon estuary. J. Fish Biol. 76(3):467-486. PMid:20666891. http://dx.doi.org/10.1111/j.10958649.2009.02487.x

WHITFIELD, K.A. 1999. Ichthyofaunal assemblages in estuaries: a South African case study. Rev. Fish Biol. Fish. 9:151-186. http://dx.doi. org/10.1023/A:1008994405375 